\title{
Les communications de paralangage dans les organisations ou la para-communication comme phénomène organisational majeur
}

\section{Alex Mucchielli}

\section{(2) OpenEdition}

1 Journals

Édition électronique

URL : https://journals.openedition.org/communicationorganisation/2451

DOI : 10.4000/communicationorganisation.2451

ISSN : $1775-3546$

Éditeur

Presses universitaires de Bordeaux

Édition imprimée

Date de publication : 1 novembre 2000

ISSN : 1168-5549

\section{Référence électronique}

Alex Mucchielli, « Les communications de paralangage dans les organisations ou la paracommunication comme phénomène organisational majeur », Communication et organisation [En ligne], 18 | 2000, mis en ligne le 27 mars 2012, consulté le 05 août 2021. URL : http:// journals.openedition.org/communicationorganisation/2451 ; DOl : https://doi.org/10.4000/ communicationorganisation.2451

Ce document a été généré automatiquement le 5 août 2021

(C) Presses universitaires de Bordeaux 


\title{
Les communications de paralangage dans les organisations ou la para- communication comme phénomène organisational majeur
}

\author{
Alex Mucchielli
}

\section{Introduction}

1 Prendre un point de vue « communicationnel » sur les organisations, c'est considérer que tout, dans une organisation, peut être considéré comme une communication.

2 Pierre de Saint-Georges proposait déjà, dans la revue Communication et Organisation, en 1993 (dans le numéro 4. novembre 1993, pp. 70-93), de prendre ce parti pris. Il faisait remarquer que « toute l'entreprise « se communique » par l'organisation et l'utilisation de l'espace, la gestion du temps, les symboles d'appartenance, les échanges verbaux et non verbaux, les accessoires professionnels, la couleur des murs, la disposition interne et externe des bâtiments, la profondeur des moquettes, les bruits, les odeurs, les toilettes, la cantine ou le restaurant,... » C'était une idée intéressante qui arrivait alors trop tôt dans le domaine des Sciences de l'information et de la communication. Depuis la discipline a fait quelques avancées en intégrant mieux les apports de l'école de Palo Alto, en élaborant une définition nouvelle des « processus de la communication » et en fournissant de ce fait une théorie sémio-contextuelle du travail de la « communication généralisée ». Cette communication généralisée étant justement tout ces éléments cités par Saint-Georges qui prennent un sens dans le contexte de l'organisation.

Nous savons mieux désormais utiliser le point de vue systémique. Nous savons que, de ce point de vue. dans une entreprise (comme ailleurs), on ne communique pas, " on prend part à une communication». Nous savons aussi qu'une communication, pour être comprise, doit être remise dans le contexte du système dans lequel elle s'insère. En généralisant, nous pouvons dire que toute «communication interne» ne peut être 
« lue » directement. Elle s'inscrit d'abord, comme le sous entendait Saint-Georges, dans les contextes de la situation de l'entreprise.

4 Je voudrais donc montrer, sur un exemple concret, en m'appuyant sur ces derniers apports, que la " para-communication » (cette communication qui se fait en dehors des paroles et des écrits) est omniprésente dans les organisations. Je serai même amené à conclure que l'attention portée à ces phénomènes de "communication implicite » (autre nom du même phénomène), va permettre de renouveler l'analyse organisationnelle pour l'instant occupée quasi exclusivement par la psychosociologie et la sociologie.

\section{Une étude de cas : une procédure d'information descendante dans une entreprise}

\section{Le contexte global}

5 Considérons le Chef de Département de production du type «Ligne produit » dans une grande entreprise multinationale de fabrication de produits pharmaceutiques.

6 Il reçoit un grand nombre d'informations en provenance de sa hiérarchie et des divers autres départements de l'entreprise. De sa hiérarchie il reçoit des informations stratégiques et générales : de la D.R.H., des informations de politique du personnel, de gestion des carrières, des règles de promotion, des postes libres au concours interne, des règles nouvelles de droit applicables à la gestion des personnels... ; du département contrôle de la qualité, il reçoit directement des instructions, des procédures... applicables immédiatement pour toutes ses unités et sous-unités de production; du Département informatique, il reçoit, de même, des instructions, règlement interne d'utilisation des ordinateurs et logiciels, des procédures d'achat, de mise en vente, de maintenance,.... à appliquer impérativement pour que les productions finales des produits soient les meilleurs possibles...

7 Le cahier des charges du poste de ce chef de Département lui précise qu'il doit informer ses collaborateurs et tous les personnels de son département, au sujet de toutes les informations nécessaires à l'exécution la plus parfaite possible des tâches aboutissant à la production finale des médicaments ou composants qui sortent de son département.

8 Pour ce faire, le chef de Département a institué avec ses chefs de services (une quinzaine) une réunion d'information mensuelle. Il a, en outre, instauré, aux échelons qui sont en dessous de lui, le même type de réunions. Ceci veut dire que ses chefs de service font de même, tous les mois, avec leurs chefs d'unité et que les chefs d'unité font de même, tous les mois, avec leurs équipes de production. Le chef de Département a informé sa direction de cette manière de procéder. La direction a tacitement validé cette procédure d'information descendante.

\section{Le déroulement concret des réunions d'information descendante}

9 En ce qui concerne le chef de Département, ses cadres sont prévenus que la réunion d'information se tient de 10 à 12 heures tous les premiers mardis du mois en salle de réunion. Ils ont l'instruction formelle d'y participer. 
10 Tous les premiers mardis du mois, vers $10 \mathrm{~h} \mathrm{10-10} \mathrm{h} 15$. le chef de Département arrive, tout essoufflé à la réunion d'information. Ses cadres arrivent, comme lui, en courant, entre $10 \mathrm{~h} 05$ et $10 \mathrm{~h} 15$. Après un rapide échange de poignées de mains avec les cadres présents, le chef de Département commence la lecture des informations à transmettre à tous qui lui sont parvenues. Pour ce faire, il ouvre un grand dossier rouge qui contient, par ordre d'arrivée, les fax, notes de service, tirages d'Email, circulaires, photocopies d'écran,... contenant les diverses informations indispensables à l'effection du travail et en provenance des diverses sources que nous avons signalées. La séance est longue et fastidieuse. Il lit et commente oralement, quelquefois, une information qui lui paraît plus importante que les autres. Les cadres écoutent en silence, dans une attitude qui montre aussi un certain détachement. Ils ne prennent pas de notes et se contentent d'opiner de la tête lorsque le chef les regarde. Très souvent, la réunion « déborde » sur l'horaire prévu et quelques cadres font comprendre au chef, en regardant leur montre, qu'il est temps de finir parce qu'ils ont d'autres réunions importantes qui les attendent. Quelques-unes d'ailleurs se lèvent et quittent discrètement la réunion vers $12 \mathrm{~h}$ 05-12hlo. Le chef du Département met fin à sa réunion, assez souvent sans avoir pu lire toutes les informations de son dossier. Il conclut par ces mots : « de toute façon, vous trouverez le relevé de toutes ces informations dans le compte rendu de la réunion que Mme X. ma secrétaire, fera comme d'habitude. Avec les références qui seront portées en face de l'information, vous pourrez aller la chercher facilement dans la banque d'informations du Département à laquelle vous avez accès directement de votre ordinateur de travail personnel ».

11 Paracquit de conscience, juste avant de lever la séance, le chef de Département demande à ses cadres s'ils ont des questions à poser, des demandes de précision à formuler ou des remarques à faire sur telle ou telle information. Aucun cadre n'intervient jamais à ce moment pour faire des remarques ou demander quoi que ce soit.

12 Ce qui se passe, et que nous venons de décrire au niveau du Département, au sujet de ces informations descendantes, se déroule de la même maniere, aux niveaux inférieurs. Les chef de service font le même type de réunions avec leurs chefs d'unité et les chefs d'unite font de même avec leurs équipes. Ainsi dans l'entreprise, les exigences tonnelles de la direction générale de «faire passer l'information" sont respectées. Mais personne n'est satisfait et tout le monde critique la procédure «qui ne permetpas de voir clair dans la masse d'informations que l'on reçoit». Mais remarquons aussi, que personne, à quelque niveau que ce soit, n'intervient pour proposer des modifications des manières de faire.

\section{L'étude « communicationnelle » du eus : l'analyse des communications implicites}

13 Pour faire le type d'étude que je propose, nous devons nous demander ce que veulent dire, dans le contexte de l'entreprise, les manières de faire concrètes (les conduites) du chef de Département et de ses cadres dans la préparation et la tenue de ces réunions d'informationdescendantes

14 La masse d'informations a gérer est une des données intangibles et caractéristiques de cette entreprise. La bonne production finale d'un produit pharmaceutique exige l'integration de quantité de contraintes stratégiques (marketing, compétition entre 
firmes,...), de contrôle de qualité (législation, procédures de certification,...). informatiques (cohérence des matériels et logiciels dans un groupe multinational...).... C'est donc un des problèmes spécifiques et importants de management et du management dans une telle structure organisationnelle.

Or on constate que ce problème n'est pas traité en tant que problème, par le management. On se contente de la définition d'une procédure très générale et vague de diffusion des informations: des réunions mensuelles, hiérarchie-subordonnés. Une telle non préoccupation portée à ce problème est une conduite collective du management. En tant que conduite, elle est donc une communication. Cette communication prend un sens dans le contexte global de l'entreprise. Cela veut dire qu'elle délivre un «message » à tous les personnels de l'entreprise. Les personnels, en effet, ne peuvent pas ne pas décoder (même à un niveau non explicite) cette manière collective de la hiérarchie de se comporter face au problème de la masse d'informations à gérer. Le "message " diffus qui passe donc est du genre "pour bien manager, la gestion de l'information est secondaire", ou "l'information est un problème secondaire »... Ce message est d'ailleurs repris et amplifié par toute la ligne hiérarchique.

Nous pouvons faire, par ailleurs, d'autres constats. Nous allons aussi essayer de les «faire parler ». Le cas nous rapporte qu'il n'y a qu'une seule réunion par mois pour gérer correctement la masse d'informations. Que le chef de Département arrive, en retard, essoufflé. Il communique par là. qu'il avait d'autres choses importantes à faire. Il livre "en vrac", à ses cadres, le contenu des papiers entassés dans un dossier. Il communique par là qu'il n'a pas pris le temps, le soin de préparer sa réunion, en classant les informations à l'aide d'une grille adaptée. Ce travail étant "secondaire " par rapport aux autres tâches qu'il a à faire. Il lit les informations et n'exige pas de ses cadres qu'ils prennent des notes ou qu'ils commentent réellement l'impact pour eux de telle ou telle information. Il « renvoie » tout le monde en fin de réunion à la lecture d'un P.V. et à la consultation d'une B.D. Il semble dire qu'il n'est là « que " pour faire ce que la direction lui demande formellement : transmettre l'information. Sa conduite peut même, dans une certaine mesure, être comprise comme une critique des exigences de la hiérarchie, voire même comme un discret sabotage de ces exigences car, sa manière de faire ne rend pas efficace la transmission des informations

On peut dire que ce sont tous ces «messages implicites » que ses cadres reçoivent. Il faut croire qu'ils les comprennent bien et qu'ils sont d'accord puisque, eux-mêmes font pareil avec leurs subordonnés : ils reproduisent (imitent) les conduites de leur chef et donc diffusent cette "communication implicite» (mise au second plan, par la ligne hiérarchique, du problème de la gestion de la masse d'informations).

C'est d'ailleurs un peu ce qui commence à se passer, dans cette entreprise, lorsque toutes les informations à intégrer par les cadres sont « renvoyées » à un compte rendu, à des numéros d'inventaire d'une banque de données informatique. Comme si la technique informatique, la mise en stock des informations, allait résoudre le problème caché et important qui est non pas le stockage et l'accès aux informations, mais leur intégration et utilisation par toute la hiérarchie. 


\section{Des conséquences pour l'audit communicationnel et l'intervention}

département aux services fonctionnels qui lui «balancent» des informations (ceci étant sa perception des choses). Cette passivité (qui est donc, pour nous, aussi une para communication) est-elle perçue comme une "acceptation", une "soumission", un " dédain ", une "mise à distance ", un "rejet » ou autre chose encore. Toutes ces interprétations sont possibles de la part des chefs de département des services fonctionnels. Chacun va lui donner un sens (la «lire»), à partir des contextes normatifs, de positionnement et relationnel qu'il privilégie (les contextes de la situation qui définissent, pour lui, la situation des relations de travail dans l'entreprise).

5 Pour le département "contrôle qualité ", il est évident que ce département de production est « rétif au contrôle qualité : la preuve, c'est qu'il ne répond jamais aux procédures nouvelles qu'on lui fait parvenir et que si l'on va dans les unités, contrôler quelques applications, on découvre des cadres «qui font semblant de ne pas être au 
courant » et l'on est obligé d'aller avec eux dans leur propre banque de données pour voir que la procédure y est bien (!).

Pour le département "stock en matières premières ", ce département de production est complètement sous le stress de ses préoccupations de productions et des risques d'erreurs de la fabrication. Il est donc incapable d'intégrer directement les contraintes de la gestion des stocks de matières premières et de la rationalisation en cours de cette gestion. Certes, il faut toujours les informer officiellement et hiérarchiquement des procédures à respecter mais l'on sait que cela ne sert à rien. Aussi se comporte-t-on concrètement autrement avec eux. On organise, dans leurs locaux, aux heures qui leur conviennent, des réunions avec les chefs d'unités. Le chef de département «stock en matières premières" et/ou ses principaux collaborateurs se déplacent pour aller rapidement informer ces cadres des nouvelles procédures et discuter avec eux des conséquences pour eux. Les chefs de service du département production sont au courant et sont satisfaits de cette manière de faire.

Que voyons-nous d'après les rapides résultats d'enquête? Que la non-communication de ce Département de production appelle en retour des actions de ses partenaires qui ne sont pas sans répercussion sur sa propre marche. La non-maittrise managériale des informations (avec la non-réponse qui va avec), affecte le fonctionnement du département. Celui-ci croyant éviter des perturbations en faisant le « gros dos » ne fait que provoquer des interventions plus fortes de ses partenaires.

Le "contrôle qualité " va redoubler de vigilance et renforcer les contraintes de ses procédures qualité pour ce département « rétif; par ailleurs, le Département «stock matières premières " va finir par imposer discrètement ses façons de voir à ce département de production alors qu'en partenaire, il devrait aussi tenir compte des contraintes de la ligne de production. Croyant se protéger, le management de la ligne production ne fait qu'accroître les problèmes de management interne.

Si l'on se proposait "de rendre efficace la communication interne" dans cette entreprise, on voit que ce n'est qu'à partir d'une analyse complète et pertinente de la "situation communicationnelle " que l'on pourrait prétendre proposer des remédiations qui soient réellement des remédiations. Si l'on ne comprend pas les ressorts organisationnels profonds de ce qui se passe pour les acteurs, on ne peut prétendre mettre au point de «meilleurs façons de faire». On ne fait que déplacer le problème sans le résoudre.

30 Ainsi, toute solution proposée doit prendre le mal "à sa source", c'est-à-dire considérer le problème de fond de ces réactions collectives de la ligne hiérarchique production face au problème de la gestion de la masse d'information. Le problème de fond, c'est qu'il s'agit là d'un vrai problème de management qui concerne l'ensemble de la hiérarchie. Il nécessite donc d'abord une prise de conscience collective et une implication collective. Sans doute peut-on prévoir que toute "solution" qui ne passe pas d'abord, en utilisant différents moyens, par cette prise de conscience et cette implication collective soit vouée à l'échec. Les « solutions " proposées ne peuvent être qu'à base de "communication collective ». Communication permettant à la ligne hiérarchique de "construire collectivement» son analyse (sa représentation de la situation) et de « construire collectivement » les moyens de faire face (ce qui aurait du sens pour elle dans cette situation). 


\section{Conclusion}

communications - que j'ai esquissée ci-dessus, porte en elle, d'après moi, de fortes potentialités pour renouveler l'analyse organisationnelle trop exclusivement encore sous l'influence dominante de la psychosociologie et de la sociologie des organisations. La « lecture communicationnelle » des organisations que je propose, permet une autre approche. Elle permet de retrouver l'étude des phénomènes liés à la genèse du sens, ce sens (ou ensemble de significations) qui a toujours été le déclencheur des actions humaines. Elle est donc plus générale, par exemple, que la seule lecture des phénomènes organisationnels en termes de «lutte pour le pouvoir ». Elle devrait intéresser les chercheurs en communication travaillant dans le domaine organisationnel. 


\section{AUTEUR}

\section{ALEX MUCCHIELLI}

Université Montpellier 3 